\title{
ACERCA DEL STATUS ONTOLÓGICO DE LAS ENTIDADES QUÍMICAS: EL CASO DE LOS ORBITALES ATÓMICOS
}

\author{
MARTÍN LABARCA \\ CONICET/Universidad Nacional de Quilmes \\ OLIMPIA LOMBARDI \\ CONICET/Universidad de Buenos Aires
}

\begin{abstract}
The aim of the present paper is to analyze the problem of the relationship between chemistry and physics, by focusing on the widely discussed case of the atomic orbitals. We will begin by remembering the difference between the physical and the chemical interpretation of the concept of orbital. Then, we will refer to the claim made in 1999 that atomic orbitals have been directly imaged for the first time. On this basis, we will analyze the problem from a new approach, by comparing the concept of orbital used in physics with the concept of orbital used in chemistry. Such an analysis will allow us to argue for an ontological pluralism that admits the coexistence of different ontologies without priorities or metaphysical privileges. From this philosophical framework, the concepts of chemical orbital and physical orbital correspond to two different ontologies. As a consequence, chemical orbitals are real entities belonging to the ontology of molecular chemistry, and can be observed like any other entity not belonging to the quantum mechanical ontology.
\end{abstract}

Keywords: Chemistry, atomic orbital, reductionism, realism, ontological pluralism.

\section{Introducción}

El problema de la relación entre química y física es el tópico de mayor madurez en la filosofía de la química contemporánea. Tradicionalmente, este problema ha sido discutido en el ámbito epistemológico sobre la base del análisis del concepto de reducción y su aplicación en las ciencias químicas. En este ámbito, entonces, existe acuerdo entre los filósofos de la química contemporáneos en rechazar la reducción epistemológica del mundo químico al mundo de la física de las partículas elementales: las leyes y conceptos de la química no pueden expresarse adecuadamente en el lenguaje mecánico-cuántico (cfr. por ejemplo, Primas 1983, Scerri y McIntyre 1997, Vemulapalli y Byerly 1999, van Brakel 2000a, Scerri 2004).

Sin embargo, las discusiones en el ámbito ontológico han tenido poca o ninguna influencia en la controversia: hasta hace poco tiempo, existía un amplio acuerdo respecto de que las entidades químicas no son más que entidades físicas (cfr., por ejemplo, Kemeney y Oppenheim 1956, Scerri y McIntyre 1997, Vemulapalli y Byerly

Principia 14(3): 309-333 (2010).

Published by NEL — Epistemology and Logic Research Group, Federal University of Santa Catarina (UFSC), Brazil. 
1999, Luisi 2002). De este modo, la autonomía de la química como disciplina científica se respaldaba en el rechazo de la reducción epistemológica, pero admitiendo la reducción ontológica.

Durante los últimos años, el supuesto de la reducción ontológica ha comenzado a ser cuestionado desde distintos marcos filosóficos. La perspectiva incipiente de un pluralismo ontológico comienza a ganar terreno en este ámbito de discusión (Crasnow 2000, van Brakel 2000a y 2003, Scerri 2000a y 2006). En particular, en trabajos previos (Lombardi y Labarca 2004, 2005 y 2006) hemos argumentado que la autonomía de la química debe defenderse desde un marco filosófico que permita rechazar no sólo la reducción epistemológica, sino también la reducción ontológica. Esta perspectiva, inspirada en la filosofía kantiana, conduce a un pluralismo ontológico que proporciona a la ontología química el mismo status que a la ontología física.

El objetivo del presente trabajo consiste en analizar el problema de la relación entre química y física concentrando la atención en un caso particular, ampliamente discutido en la actualidad: el caso de los orbitales atómicos. Para ello, comenzaremos por recordar la diferencia en la interpretación que se brinda al concepto de orbital desde la química y desde la física, enfatizando la situación de confusión conceptual que ello genera. Mencionaremos también el acalorado debate desencadenado por la supuesta observación de orbitales atómicos. Sobre esta base, analizaremos el problema desde una nueva perspectiva, no ya centrada en las dificultades para describir átomos multielectrónicos, sino basada en la comparación de los conceptos de orbital usados en física y en química. Dicho análisis nos permitirá, finalmente, argumentar en favor de un pluralismo ontológico que admite la coexistencia de diferentes ontologías, sin prioridades ni privilegios metafísicos. Desde esta perspectiva, los conceptos de orbital químico y de orbital físico corresponden a dos ontologías diferentes, ambas de igual status ontológico, cada una de las cuales posee una existencia autónoma fundada en la fecundidad de la disciplina científica correspondiente.

\section{El marco de la discusión: la relación entre química y física}

Hasta principios del siglo XX, la química siguió un desarrollo histórico independiente de la física: los fenómenos químicos eran concebidos en su propia especificidad y con sus propias regularidades (cfr. Nye 1993). Sin embargo, con el arrollador éxito predictivo de la mecánica cuántica, durante el siglo XX la química queda marcada a fuego por el famoso dictum de Dirac, según el cual "Las leyes físicas fundamentales necesarias para la teoría matemática de una gran parte de la física y la totalidad de la química [son] completamente conocidas desde la mecánica cuántica" (Dirac 1929, p.714). Aún en la actualidad, esta perspectiva continúa siendo ampliamente adop-

Principia 14(3): 309-333 (2010). 
tada en el seno de la comunidad científica (cfr. por ejemplo, Wasserman y Schaefer 1986, Bader 2003). Desde luego, este enfoque reductivo atenta contra la autonomía de la química como disciplina científica o, al menos, la coloca en una posición subordinada respecto de la física: mientras que la física sería la ciencia "fundamental" que representa la realidad tal como es en sí misma, la química resultaría una disciplina meramente "fenomenológica" que sólo describe los fenómenos tal como se nos presentan.

Durante los últimos años, muchos filósofos de la química han comenzado a desafiar este enfoque tradicional con el propósito de recuperar la autonomía de la química y, a fortiori, la legitimidad de la filosofía de la química. En algunos casos, la autonomía de la química como disciplina es defendida en términos históricos, enfatizando las diferentes tradiciones que marcaron la evolución histórica de la física y de la química (cfr. Vancik 1999). Sin embargo, la línea de argumentación más frecuente es la que enfatiza la imposibilidad de reducción epistemológica de la química a la física. Si bien los argumentos particulares difieren entre sí, los autores concuerdan en considerar que las descripciones y los conceptos químicos no pueden derivarse de los conceptos y las leyes de la física, tal como lo supone el reduccionismo epistemológico tradicional (cfr., por ejemplo, van Brakel 2000a, Cap.5). En este sentido, Vemulapalli y Byerly (1999) sostienen que la reducción epistemológica falla incluso en casos relativamente sencillos: la relación interteórica sólo puede establecerse introduciendo datos específicamente químicos. Desde una perspectiva similar, Scerri y McIntyre concluyen que deberíamos "renunciar a reducir epistemológicamente la química a la física" (Scerri y McIntyre 1997, p.220).

Sin embargo, a pesar del rechazo de la reducción epistemológica, la mayoría de los filósofos de la química siguen aferrados a la idea de una reducción ontológica de las entidades químicas a entidades físicas. Mientras algunos autores adoptan explícitamente una posición fisicalista (Vemulapalli y Byerly 1999), otros consideran que la imposibilidad de reducción epistemológica se debe exclusivamente a nuestros limitados poderes de observación y cálculo (Benfey 2000, Luisi 2002). De este modo, si bien se preserva la autonomía de la química como disciplina científica, la reducción ontológica confina el mundo de la química al reino de lo meramente aparente o ilusorio: la realidad independiente estaría poblada sólo por las entidades y propiedades de la física fundamental; por el contrario, la forma o estructura molecular no sería más que una "poderosa e iluminadora metáfora" (Woolley 1982, p.4).

En un ambiente dominado por las discusiones epistemológicas, algunos autores han abordado la cuestión ontológica sobre la base de alguna forma de emergentismo que rechaza la reducción ontológica del mundo químico: las entidades y propiedades químicas son objetivamente existentes puesto que "emergen" a partir del nivel físico fundamental. Ya Broad (1925) proponía la noción de emergencia apelando a ejemplos de la química, tales como la emergencia del cloruro de potasio. Actualmen- 
te, el recurso a las nociones de emergencia o superveniencia reaparece en diversos autores. Por ejemplo, Bunge (1982) considera que la composición es una propiedad emergente que varía en el tiempo. Del mismo modo, Scerri y McIntyre (1997) se preguntan si la química superviene a la física analizando, en particular, la propiedad 'olor'. Por su parte, Hendry (2006) evalúa la posibilidad de causación descendente en química, mientras que Bishop (2005) considera que una adecuada elucidación del concepto de emergencia permite vincular apropiadamente la mecánica cuántica y la química molecular.

En general, estas posiciones de corte emergentista se basan en un monismo fisicalista no reductivo que, no obstante, continúa admitiendo la prioridad ontológica del mundo físico respecto de los niveles emergentes. Por lo tanto, si bien el emergentismo no confina las entidades y propiedades químicas al reino de lo meramente ilusorio o "metafórico", las dota de una existencia ontológicamente secundaria, en tanto derivada de la existencia de los ítems físicos. En otras palabras, se continúa estableciendo una relación de dependencia ontológica del dominio químico respecto del físico. Precisamente en este sentido Scerri y McIntyre (1997, p.18) consideran que "la dependencia ontológica de la química respecto de la física parece ser casi un resultado inevitable". En consecuencia, si bien el emergentismo no priva a la química de un ámbito de estudio propio, ubica a la disciplina en un lugar "secundario" respecto de la física: mientras que la química se ocupa de entidades y propiedades derivadas o dependientes, la física estudia el nivel "fundamental" y más profundo de la realidad, cuya prioridad ontológica es aquello que brinda existencia objetiva al nivel emergente de la química. De este modo, continúa admitiéndose la tradicional jerarquía de las ciencias naturales, que ubica a la física en el lugar de privilegio y considera a la química como una "ciencia secundaria".

En artículos recientes (Lombardi y Labarca 2004, 2005) hemos abordado el problema de la relación entre química y física desde una perspectiva estrictamente ontológica, sobre la base de un realismo pluralista de raíces kantianas inspirado en el internalismo de Putnam (1981). Según esta posición filosófica, debemos abandonar los sueños metafísicos de omnisciencia: no existe la perspectiva del Ojo de Dios que nos permitiría conocer la realidad independiente, tal como es en sí misma. El objeto de conocimiento está siempre constituido como una síntesis entre la realidad independiente y el esquema conceptual que aporta el sujeto. Pero, a diferencia de Kant, quien ubica al sujeto y su sistema de categorías en el ámbito de lo trascendental, nuestra posición reconoce un sujeto histórico y culturalmente situado que accede a la ontología resultante de la contribución de su propio esquema conceptual.

No nos detendremos aquí en los detalles de esta posición filosófica (para ello, cfr. Lombardi y Pérez Ransanz 2011a, 2011b), sino en su aplicación al problema que nos ocupa. El pluralismo ontológico de inspiración kantiana ha jugado su papel más relevante en torno al problema diacrónico del cambio teórico, especialmente en relación 
con las revoluciones científicas, donde se ha esgrimido sobre todo contra el realismo metafísico de corte cientificista, según el cual la evolución de la ciencia converge hacia la descripción de los componentes últimos de la realidad. Sin embargo, en una versión sincrónica, dicho pluralismo puede ser aplicado al caso de las ontologías constituidas por teorías simultáneamente aceptadas en la comunidad científica. Es precisamente esta versión del pluralismo ontológico el que nos ha permitido defender la autonomía ontológica del mundo químico (Lombardi y Labarca 2004, 2005). En efecto, una vez que reconocemos que no existe un mundo "ya hecho" al cual tengamos acceso directo, y que cada esquema conceptual constituye su propia ontología, debemos admitir que la ontología cuántica es tan teórico-dependiente como la ontología química. Si la química pudiera reducirse epistemológicamente a la mecánica cuántica, podría esgrimirse una razón para creer en la reducción ontológica del mundo químico al mundo cuántico. Pero cuando se acepta la irreductibilidad epistemológica de la química, no hay otro motivo más que un prejuicio metafísico para suponer la prioridad ontológica del dominio físico. Las entidades químicas, como orbital o molécula, y las propiedades químicas, como quiralidad, enlace químico o estructura molecular, no necesitan referirse a propiedades físicas para adquirir legitimidad ontológica: son propiedades pertenecientes al mundo de la química, y su existencia objetiva no depende de su reducción o emergencia ontológica respecto de las propiedades supuestamente más básicas de la física, sino del hecho de ser descritas por una disciplina como la química, cuya fecundidad pragmática actualmente no puede ponerse en duda.

Nuestra aplicación del pluralismo ontológico de raigambre kantiana al problema de la relación entre química y física ha comenzado a tener repercusiones en el ámbito de la filosofía de la química. Mientras algunos autores se muestran escépticos acerca de los alcances de la autonomía ontológica propuesta (Needham 2006), otros consideran que esta posición filosófica abre un camino para abordar el problema (Scerri 2005, McIntyre 2007). Por estos motivos, intentaremos aquí enriquecer la discusión, no ya a través de argumentos generales, sino sobre la base del análisis de un caso concreto de particular relevancia para la química: el concepto de orbital atómico.

\section{El modelo atómico de Bohr}

La analogía entre el sistema solar y la estructura del átomo, con un núcleo central y electrones orbitando a su alrededor, fue discutida por primera vez por el físico francés Jean Perrin en 1901. En 1903, Hantaro Nagaoka sugería un modelo al que denominó "átomo saturniano", en el cual los electrones orbitaban alrededor del núcleo en una serie de anillos concéntricos (cfr. Scerri 2007). El célebre experimento de

Principia 14(3): 309-333 (2010). 
Lord Rutherford en 1910, donde se empleaban hojas delgadas de oro y otros metales como blanco de partículas $\alpha$ emitidas por una fuente radiactiva, respaldó el modelo "planetario" del átomo.

Mediante los incipientes conceptos cuánticos, en 1913 Niels Bohr empleó la noción de "órbitas atómicas" en su teoría del átomo de hidrógeno. Nacía el célebre átomo de Bohr: un núcleo cargado positivamente rodeado de electrones negativos dispuestos en órbitas concéntricas y capaces de "saltar" entre las distintas órbitas por emisión o absorción de energía luminosa. Sobre la base de este modelo, Bohr brindó una explicación aproximada de la disposición de los elementos en la tabla periódica apelando a las órbitas electrónicas para átomos multielectrónicos (para mayores detalles, cfr. Scerri 2007).

Si bien el modelo atómico de Bohr fue un eslabón histórico muy importante para el desarrollo de la mecánica cuántica, pronto comenzó a mostrar inconsistencias teóricas cuando se lo aplicó, entre 1913 y 1925, a átomos multielectrónicos y a moléculas. La formulación definitiva de la mecánica cuántica a fines de la década de 1920, de la mano de Schrödinger, Heisenberg, Born y von Neumann entre otros, modificó sustancialmente la concepción inicial de los electrones en el átomo: ahora los electrones se disponían alrededor del núcleo en tres dimensiones. Además, según el principio de indeterminación de Heisenberg, los electrones dejaron de concebirse orbitando alrededor del núcleo en trayectorias definidas: se comienza, así, a hablar de "orbitales" en lugar de órbitas.

Al imponerse definitivamente la mecánica cuántica en la comunidad científica, los físicos acabaron abandonando el modelo "pictórico" del átomo para adoptar una descripción puramente formal en términos de los elementos teóricos de la teoría cuántica. Los químicos, en cambio, retuvieron la idea de electrones en torno al núcleo en ciertas disposiciones espaciales que permitían explicar los enlaces químicos y la forma molecular. De este modo, se produce la ruptura conceptual entre la descripción física y la descripción química del átomo.

\section{La descripción cuántica del átomo}

La descripción física del átomo se basa en la ecuación fundamental de la mecánica cuántica, la ecuación de Schrödinger, que en su versión independiente del tiempo se expresa como:

$$
H \Psi=E \Psi
$$

donde $H$ es el operador hamiltoniano que representa la energía total del sistema, $E$ representa los valores de la energía total, autovalores de $H$, y $\Psi$ es la función de onda que representa el estado del sistema y que, en el caso de la descripción de los electrones en el átomo, se expresa en coordenadas espaciales.

Principia 14(3): 309-333 (2010). 
Para los átomos hidrogenoides (aquéllos formados por un electrón y $Z$ protones, como el $\mathrm{H}$ o los iones $\mathrm{He}^{+}, \mathrm{Li}^{2+}$ o $\mathrm{Be}^{3+}$ ) la ecuación toma la forma:

$$
-\frac{\hbar^{2}}{2 m} \frac{d^{2} \Psi(r)}{d r^{2}}+V(r) \Psi(r)=E \Psi(r)
$$

donde $\hbar=h / 2 \pi$ (con $h$ : constante de Planck), $r$ es el vector posición del electrón, $m$ es su masa y $V(r)$ es la energía potencial coulombiana generada por el núcleo. Para este tipo de átomos, la ecuación tiene solución analítica, la cual consiste en una función de onda $\Psi$ expresada en coordenadas polares esféricas $(r, \theta, \phi)$ y caracterizada por tres números cuánticos $n, l$ y $m$ :

$$
\Psi_{n, l, m}(r, \theta, \phi)=R_{n, l}(r) \Theta_{l, m}(\theta) \Phi_{m}(\phi) .
$$

En los átomos hidrogenoides, entonces, la función de onda es factorizable en un factor radial $R_{n, l}(r)$ y un factor angular $\Theta_{l, m}(\theta) \Phi_{m}(\phi)$. El número cuántico principal $n$ proporciona los niveles de energía permitidos del electrón en el átomo; el número cuántico $l$ indica los valores permitidos del momento angular total del electrón, mientras que el número cuántico $m$ indica los valores posibles de la componente $\%$ del momento angular total.

Sobre esta base se definen los estados estacionarios posibles (autofunciones del Hamiltoniano) del electrón en un átomo hidrogenoide. Para $n=1$ y $l=m=0$, la función de onda se expresa:

$$
\Psi_{1,0,0}(r)=\sqrt{\frac{1}{\pi}}\left(\frac{Z}{a_{0}}\right)^{3 / 2} e^{-\frac{Z_{r}}{a_{0}}}
$$

donde $a_{0}$ es el radio de Bohr. En este caso la función de onda tiene simetría radial, y expresa el estado del electrón en el menor nivel de energía. Otra solución de la ecuación de Schrödinger corresponde a $n=2$ y $l=m=0$ :

$$
\Psi_{2,0,0}(r)=\frac{1}{4} \sqrt{2 \pi}\left(\frac{Z}{a_{0}}\right)^{3 / 2}\left(2-\frac{Z_{r}}{a_{0}}\right) e^{-\frac{z_{r}}{2 a_{0}}}
$$

También en este caso la función de onda tiene simetría esférica. Esta simetría se pierde en el caso $n=2, l=1$ y $m=0$, donde $\Psi$ comienza a presentar dependencia angular:

$$
\Psi_{2,1,0}(r, \theta, \phi)=\frac{1}{4} \sqrt{2 \pi}\left(\frac{Z}{a_{0}}\right)^{5 / 2} r e^{-\frac{z_{r}}{2 a_{0}}} \operatorname{sen} \theta \cos \phi .
$$

De este modo, para las diferentes combinaciones de valores de los números cuánticos $n, l$ y $m$ se obtienen las distintas funciones de onda que representan los estados estacionarios admisibles en un átomo hidrogenoide.

Principia 14(3): 309-333 (2010). 
Para átomos multielectrónicos, la solución de la ecuación de Schrödinger en su forma general es imposible por vía analítica. Por ello, para resolverla se utilizan diferentes técnicas, siendo la más corriente el método de Hartree-Fock. Este método consiste en partir del supuesto de que la función de onda del conjunto de electrones es "separable", esto es, puede expresarse como producto de las funciones de onda para cada electrón individual. Este supuesto inicial equivale a despreciar las interacciones de los electrones entre sí y considerar el estado de cada electrón como independiente de los restantes. Para tomar en cuenta los efectos medios de las interacciones, en un segundo paso se usan todas las funciones de onda electrónicas calculadas previamente, salvo una, para calcular el potencial que afecta al electrón excluido y recalcular así su función de onda. Esta nueva función de onda se agrega al conjunto de funciones que, nuevamente, se utiliza para calcular la función de onda de otro electrón. El proceso se repite hasta que no se obtiene un cambio significativo entre una iteración y la siguiente.

Si bien el método de Hartree-Fock toma en consideración los efectos promedio de las interacciones entre electrones, la función de onda resultante continúa siendo un producto de funciones de onda de electrones individuales, que ignora los efectos instantáneos de las interacciones electrónicas. Para considerar tales efectos se utilizan otras técnicas más complejas, que desembocan en la imposibilidad de adjudicar números cuánticos a los electrones individuales: la función de onda del sistema de electrones en el átomo deja de ser separable en términos del producto de los electrones componentes.

Hasta aquí hemos recordado resumidamente el modo en que se aborda actualmente la descripción del átomo en términos de la mecánica cuántica. Veamos ahora cómo la física y la química "leen" estos resultados desde sus propios marcos conceptuales.

\section{El concepto de orbital en química y en física}

La química recoge el formalismo cuántico para describir el átomo, pero retiene el concepto de orbital a través de la regla de Born, según la cual el cuadrado de la función de onda representa una probabilidad. En un sistema de $N$ partículas cuyas posiciones vienen dadas por los vectores $r_{1}, r_{2}, \ldots, r_{N}$, el estado queda representado por la función de onda $\Psi\left(r_{1}, r_{2}, \ldots, r_{N}\right)$ : según la regla de Born, el cuadrado de dicha función, $\left|\Psi\left(r_{1}, r_{2}, \ldots, r_{N}\right)\right|^{2}$, representa la probabilidad de que la partícula 1 se encuentre en la posición $r_{1}$, la partícula 2 en la posición $r_{2}, \ldots$, y la partícula $N$ en la posición $r_{N}$.

Sobre esta base, en química se concibe un orbital atómico como la región del espacio en la cual es más probable que se encuentren los electrones en un átomo (tomando, en general, la cota del 90-95\% para fijar un límite arbitrario a dicha

Principia 14(3): 309-333 (2010). 
región). Así, en los átomos hidrogenoides la función de onda $\Psi_{1,0,0}$ (ver ecuación (4)) se hace corresponder al orbital $s$ y suele designarse como $\Psi_{1 s}$; análogamente, $\Psi_{2,0,0}=\Psi_{2 s}$ (ver ecuación (5)) se hace corresponder al orbital $2 s$ y $\Psi_{2,1,0}(r)=$ $\Psi_{2 p_{x}}$ (ver ecuación (6)) se hace corresponder al orbital $2 p_{x}$. Estos orbitales son los que suelen representarse esquemáticamente mediante los conocidos diagramas que aparecen en todos los libros de texto.

Desde esta perspectiva, un orbital atómico es una región del espacio que, si bien estrictamente posee bordes difusos, tiene una forma definida. Es precisamente la estructura geométrica del orbital atómico aquello que, en química, explica el modo en que los átomos se unen entre sí a través de los enlaces químicos dando lugar a moléculas con una forma o estructura geométrica definida. A su vez, la forma o estructura molecular (molecular shape) cumple un papel central en la comprensión de ciertas propiedades macroscópicas de las sustancias, como su reactividad y sus manifestaciones en espectroscopía. La enorme utilidad teórica del concepto de orbital atómico en química explica el hecho de que, en general, los químicos sean realistas respecto del concepto, esto es, atribuyan al orbital atómico una existencia real.

En física, en cambio, el concepto de orbital ha perdido su referente objetivo. En los textos de física o de química cuántica, el orbital atómico suele ser definido como la función de onda de un átomo monoelectrónico y, por ello, se considera sólo una aproximación en átomos multielectrónicos. En consecuencia, el concepto de orbital es concebido como una mera entidad matemática en átomos monoelectrónicos, y en átomos multielectrónicos es considerado una mera herramienta cuya utilidad reside únicamente en permitir calcular el estado de los electrones en el átomo, en general a través de importantes aproximaciones. Pero, en ambos casos, el término 'orbital' estrictamente carece de referente ontológico.

La diferencia que presenta el concepto de orbital en química y en física puede parecer un detalle menor que sólo da pie a discusiones filosóficas desconectadas con la práctica científica. Sin embargo, éste no es el caso: en la medida en que el desacuerdo no apunta a una cuestión meramente epistemológica sino a un problema ontológico, el debate acerca de la existencia o inexistencia de los orbitales condujo a una tensión en el ámbito científico que no tardó en estallar.

\section{6. ¿Observación de orbitales?}

Durante el segundo semestre de 1999, una noticia sacudió al mundo de la química y de la física: los orbitales habían sido observados y fotografiados por primera vez (Zuo et al. 1999). El resultado experimental, anunciado en la tapa de la prestigiosa revista científica Nature bajo el inequívoco título "Orbitals observed", es descripto por los autores en los siguientes términos: "Todos nuestros mapas muestran fuertes distri-

Principia 14(3): 309-333 (2010). 
buciones no-esféricas de carga alrededor de los átomos de cobre, con la forma [shape] característica de los orbitales d" (Zuo et al. 1999, p.51). La "visualización" de orbitales fue rápidamente comunicada a través del mundo científico (cfr. por ejemplo, Jacoby 1999, Yam 1999, Zurer 1999) y fue nominada como uno de los cinco highlights del año en la química (Zurer 1999). A su vez, científicos de prestigiosas universidades señalaron de inmediato la utilidad de este trabajo experimental como punto de partida para la comprensión de otros fenómenos, como la superconductividad de óxidos de cobre a altas temperaturas (cfr. Humphreys 1999). Algunos libros de texto no tardaron en reproducir las fotografías obtenidas en los experimentos, como confirmación experimental de las predicciones teóricas (Tinoco Jr. et al. 2002). Mientras tanto, la visualización de orbitales en otro compuesto fue comunicada por otro grupo experimental poco después de la publicación del primer artículo (Pascual et al. 2000).

A pesar del impacto y la expectativa que generó el resultado de la observación de orbitales, algunos químicos y filósofos de la química objetaron rápidamente las conclusiones obtenidas por los investigadores responsables de la experiencia, señalando el supuesto error conceptual cometido en la interpretación de las visualizaciones (Scerri 2000b, 2001, Wang y Schwarz 2000a). Por ejemplo, Eric Scerri insistió en que los orbitales no pueden ser visualizados, no sólo porque las funciones de onda no son observables, sino porque, desde la perspectiva de la mecánica cuántica, los orbitales son sólo ficciones matemáticas desprovistas de cualquier existencia real. El error conceptual, señala el autor, consiste en confundir el concepto de orbital con el concepto de densidad de carga (o de densidad de electrones) el cual, efectivamente, sí se observa durante los experimentos. Es interesante señalar que esta observación conceptual le es concedida a Scerri por el grupo de investigadores que publicó el artículo original en Nature (Spence et al. 2001).

Sin embargo, algunos educadores en química han reaccionado frente a este enfoque normativista, señalando que la interpretación no realista tendrá muy poco impacto en la utilización de los orbitales en la enseñanza de la disciplina (Richman 1999a, b, Emerson 1999). A partir de ésta y otras críticas, Scerri comenzó a mitigar su posición, adoptando una "posición intermedia" entre normativismo y naturalismo: si bien los orbitales no pueden ser observados de acuerdo con la mecánica cuántica, los educadores pueden emplearlos de manera realista, pero señalando cuidadosamente sus limitaciones (Scerri 2006). Cuando el debate parecía concluir, se anunció, recientemente, la visualización de orbitales moleculares del nitrógeno en fase gaseosa (Itatani et al. 2004).

Si hasta el momento de las primeras noticias la discrepancia acerca del significado del término 'orbital' podía continuar confinada al plano epistemológico, con el anuncio de la observación de orbitales la discusión ingresó decididamente en el terreno ontológico. Ya no se trata de establecer el papel explicativo del concepto de

Principia 14(3): 309-333 (2010). 
orbital o la posibilidad de su descripción en términos mecánico-cuánticos: ahora el problema se convierte en la cuestión de decidir si los orbitales existen o no.

Como hemos visto, Eric Scerri adopta una posición clara respecto del problema. Según el autor, los experimentos comunicados como visualización de orbitales han sido incorrectamente interpretados puesto que los orbitales no son observables. Y tal inobservabilidad no depende de limitaciones empíricas sino del simple hecho de que el término 'orbital' carece de referencia: estrictamente, los orbitales no existen.

Los motivos de Scerri (2001) para tan drástica conclusión se apoyan en dos líneas argumentativas. El primer argumento parte de recordar las aproximaciones necesarias para la descripción de átomos multielectrónicos en términos de los orbitales de los electrones individuales: puesto que en estos casos la función de onda no es factorizable en las funciones de onda de los electrones componentes, estrictamente no es posible adjudicar números cuánticos definidos a cada electrón en forma independiente. Por ello, Scerri considera que la utilidad de los orbitales consiste únicamente en brindar una base en la cual la función de onda se expresa matemáticamente, con el grado de precisión permitido por el poder de cómputo disponible al momento (Scerri 2001, p.S79).

El segundo argumento, considerado por el propio autor como el más decisivo, se basa en el hecho de que, según la mecánica cuántica, los electrones carecen de una trayectoria definida: su función de onda no describe su trayectoria sino que permite calcular una distribución de probabilidad. En consecuencia, si se admite que un orbital es una función de onda, entonces los orbitales son inobservables en principio, puesto que la función de onda es una magnitud imaginaria (que incluye el número imaginario $i=\sqrt{-1}$ ). Sobre esta base, Scerri concluye que "sólo puede observarse el cuadrado de un orbital atómico y no el orbital atómico mismo" (Scerri 2001, p.S87).

Ambos argumentos, formulados también por otros autores (Wang y Schwarz 2000a), apuntan en la misma dirección: impugnar la existencia efectiva de los orbitales atómicos. Sin embargo, puesto que parten de premisas diferentes, cada uno de ellos exige su propio análisis.

\section{El argumento computacional}

El primer argumento se basa en una limitación matemática. En sentido estricto, sólo el átomo como un todo tiene un estado estacionario bien definido, representado por su función de onda (Scerri 2001, p.S80); dicho estado es el resultado de la interacción entre los distintos componentes del átomo, esto es, entre el núcleo y cada uno de los electrones y entre los electrones mismos. El hecho de que la ecuación de Schrödinger no pueda resolverse analíticamente para átomos multielectrónicos

Principia 14(3): 309-333 (2010). 
pone de manifiesto que, en este caso, no es posible factorizar la función de onda del átomo como producto de las funciones de onda de los electrones componentes: toda descomposición de este tipo es una mera aproximación. Puesto que no es posible, entonces, asignar una función de onda a cada electrón individual, "no podemos considerar que los electrones se encuentran en estados estacionarios. Debido a ello, no podemos asignar electrones indistinguibles a 'orbitales' individuales, como suele hacerse" (Jenkins 2003, p.1053). De aquí se concluye que "los orbitales atómicos [...] son estrictamente no referenciales ya que no selecciona ninguna entidad de la cual pueda decirse que existe físicamente en el mismo sentido en que una órbita planetaria existe" (Scerri 2001, p.S84).

Esta última afirmación de Scerri es particularmente interesante porque sugiere de inmediato la analogía con el problema clásico de los tres cuerpos. Como es bien sabido en mecánica clásica, en un sistema de $n$ cuerpos, con $n \geq 3$, que interactúan gravitacionalmente entre sí, las ecuaciones de movimiento no tienen solución analítica puesto que son no-separables. Por ejemplo, en el sistema solar, estrictamente no se puede adjudicar una ecuación de movimiento independiente a cada planeta, salvo bajo la aproximación basada en despreciar las interacciones gravitatorias de los planetas entre sí. Pero como aquí la cuestión en disputa no es epistemológica sino ontológica, el problema consiste en decidir si esta limitación matemática implica la inexistencia de las órbitas planetarias. Por supuesto, si por 'órbita planetaria' se entiende los puntos del espacio que ocuparía el planeta en su movimiento en ausencia de los demás planetas que interactúan con él, es claro que en el sistema solar las órbitas no existen y son sólo ficciones matemáticas útiles para los cálculos. Pero esta interpretación del término no sólo "ontologiza" una limitación matemática, sino que nos deja sin un nombre para designar la trayectoria que efectivamente describe el planeta, trayectoria cuya existencia no puede cuestionarse en mecánica clásica. Si, como parece más razonable, utilizamos el término 'órbita planetaria' para referirnos a la trayectoria que describe el planeta como consecuencia de su interacción con los restantes cuerpos del sistema, la no-separabilidad del sistema de ecuaciones de movimiento no implica la inexistencia de las órbitas planetarias.

Claro está que no se trata aquí de adoptar un modelo planetario del átomo ni de afirmar que los electrones poseen trayectorias definidas: el argumento debe comprenderse por analogía. Así como la no-separabilidad de las ecuaciones de movimiento clásicas no implica la inexistencia de las órbitas planetarias, la noseparabilidad de la función de onda del átomo no implica la inexistencia de los orbitales entendidos como las regiones del espacio donde es más probable que se encuentre cada electrón. Como en el caso clásico de los $n$ cuerpos, donde el cálculo en términos de trayectorias planetarias independientes es una mera aproximación, en el caso atómico la factorización de la ecuación de onda del átomo también lo es: estrictamente, la región donde es más probable que se encuentren los electro- 
nes en torno al núcleo debe calcularse con la función de onda completa. Y, como el propio Scerri admite, en un átomo multielectrónico la forma de tal región no será la característica de los orbitales de los electrones independientes, sino que "corresponderá a una forma (shape) compuesta debida a las contribuciones de las formas de todos los orbitales" (Scerri 2001, p.S82). Por lo tanto, no debería sorprendernos que, en algunos casos, las aproximaciones no sean adecuadas, puesto que los cálculos en términos de las funciones de onda de los electrones individuales "no predicen los resultados experimentales con una precisión suficiente para esos casos particulares" (Jenkins 2003, p.1059). Pero así como el hecho de que las trayectorias planetarias en torno al sol tengan formas complejas debido a la interacción entre planetas no implica la inexistencia de las órbitas planetarias, el hecho de el orbital atómico tenga una forma compleja debido a la interacción entre electrones no implica la inexistencia de la región del espacio donde es más probable que se encuentren los electrones del átomo considerados en su conjunto. La importancia de esto último reside en que precisamente en términos de la forma de esta región se explican los enlaces entre átomos y la estructura geométrica de las moléculas.

Si revisamos la reconstrucción del argumento computacional presentado al comienzo de esta sección a la luz de las consideraciones anteriores, vemos que dicho argumento rechaza la existencia de orbitales sólo en los átomos multielectrónicos, ya que sólo en estos casos la función de onda del átomo no es factorizable y no es posible adjudicar números cuánticos definidos a los electrones individuales. Por ello Scerri afirma que "el término científico 'orbital' estrictamente es no-referencial, con la excepción de cuando se lo aplica al átomo de hidrógeno o a otros sistemas monoelectrónicos" (Scerri 2001, p.S79). Pero, como hemos señalado, esta conclusión se basa en identificar una limitación matemática con una restricción ontológica. Si el concepto de orbital carece de referente porque los orbitales no existen, tal inexistencia debería afectar a cualquier tipo de átomos. Como veremos a continuación, cuando el problema se plantea no en términos de cálculo matemático sino en términos conceptuales, esto es efectivamente lo que sucede: el concepto de orbital adquiere significados totalmente diferentes en física y en química.

\section{El concepto de orbital en mecánica cuántica y en química molecular}

Tomemos el caso más sencillo de átomo monoelectrónico: el átomo de hidrógeno. ¿Qué es un orbital en este caso? En el contexto de la mecánica cuántica, el orbital atómico del átomo de hidrógeno es la función de onda de su único electrón, un ítem matemático que no posee un referente ontológico directo. En la química molecular, en cambio, el orbital atómico del átomo de hidrógeno es la región el espacio don- 
de es más probable que se encuentre el electrón en torno del núcleo, una entidad física que puede definirse (si bien en átomos multielectrónicos no calcularse) con precisión. Mientras que en el primer caso el término 'orbital' carece de referente y puede afirmarse que el orbital del átomo de hidrógeno no existe, en el segundo caso el orbital es una región del espacio que permite dar cuenta, por ejemplo, del enlace covalente simple entre dos átomos de hidrógeno en la molécula en términos del modo en que los respectivos orbitales se superponen en el espacio. Como vemos, no es necesario considerar átomos multielectrónicos para reconocer la profunda ruptura conceptual que subyace al problema.

En efecto, ya desde los albores de la mecánica cuántica se comprendió que la función de onda no representa una onda en el espacio físico: según el formalismo de la mecánica cuántica, una función de onda en representación espacial es una de las posibles representaciones del vector, perteneciente a un espacio de Hilbert, que corresponde al estado del sistema. A diferencia del estado clásico, el estado cuántico no brinda todas las propiedades del sistema a cada instante: por el principio de superposición y la existencia de observables incompatibles (que no conmutan), la teoría no asigna un valor definido a los observables de un sistema cuántico. Este resultado fue inicialmente interpretado como una limitación de la propia mecánica cuántica, que podría subsanarse mediante la incorporación de "variables ocultas" cuyos valores se encontraran determinados en todo instante. Pero la esperanza de recuperar una imagen clásica se vio frustrada con el teorema de Kochen y Specker (1967), que demuestra la contextualidad de la mecánica cuántica, esto es, la imposibilidad de asignar, de un modo consistente, un valor preciso a todos los observables de un sistema que se encuentra en un cierto estado cuántico. Se frustra así todo intento de construir un espacio de las fases clásico que determine todas las propiedades del sistema y, a la vez, permita reconstruir la estadística cuántica. De este modo, la mecánica cuántica pone en crisis la noción de objeto sustancial, esto es, la idea filosófica tradicional de individuo como sustancia individual en la que inhieren las propiedades y que brinda al objeto su identidad a través de los cambios. Otra característica central de la mecánica cuántica es la no-localidad, que en general impide considerar a un sistema cuántico con independencia de las correlaciones que mantiene con otros sistemas cuánticos con los que ha interactuado, aun cuando tales interacciones hayan cesado en el presente. Esta característica pone en crisis la noción tradicional de objeto local, la idea de una entidad que puede identificarse por su localización espacial definida. Estos problemas, que vienen quitando el sueño a quienes se han ocupado de la interpretación de la mecánica cuántica desde hace ya casi un siglo, ponen de manifiesto que toda imagen ontológica clásica de individuo resulta completamente inadecuada en el dominio cuántico: sean lo que sean los sistemas cuánticos, no son los objetos sustanciales y locales de la ciencia no-cuántica, descriptos por la noción filosófica tradicional de individuo.

Principia 14(3): 309-333 (2010). 
El objeto de estudio central de la química molecular, en cambio, es la molécula. La química molecular (o química 'clásica') las concibe como configuraciones tridimensionales de átomos en un espacio clásico euclídeo, unidos entre sí por enlaces químicos. El propósito fundamental de la disciplina consiste en identificar los diferentes tipos de moléculas y estudiar sus propiedades individuales y colectivas. En este contexto, las moléculas son objetos clásicos en un sentido sustancial y local: son entidades que poseen todas sus propiedades definidas en cada instante, que conservan su identidad a través de los cambios de sus propiedades y que poseen una localización espacial determinada. Una característica básica de las moléculas es su estructura o forma molecular (molecular shape), que resulta esencial en la comprensión de las propiedades físico-químicas de la materia; como afirma Wooley, la idea de estructura molecular "es el dogma central de la ciencia molecular" (Wooley 1978, p.1073). Para definir la forma molecular se adopta el supuesto de la estructura nuclear (nuclear frame), según el cual los núcleos de una molécula poseen posición y momento cinético determinados, como en mecánica clásica. En palabras de Primas: "El alfa y omega de la química molecular es la doctrina según la cual las moléculas existen como objetos individuales y que cada molécula posee una forma, caracterizada por su estructura nuclear" (Primas 1994, p.216). Pero, como ha insistido el propio Primas en muchos de sus trabajos (1983, 1987, 1994, 1998), el carácter holista, nolocal de la mecánica cuántica excluye el concepto mismo de forma molecular. Por lo tanto, para pensar la molécula como un objeto individual, con su propia identidad y su forma propia, es necesario ignorar toda correlación cuántica: "La forma de un estado molecular, por supuesto, debería no mostrar correlaciones holísticas con otras cantidades moleculares y, por tanto, debería estar definida de un modo no ambiguo y debería ser estable" (Amann 1992, p.32). Por otra parte, el estado de la molécula determina sus propiedades, tal como en la física clásica: la química molecular no incorpora la posibilidad de superposiciones; Amann (1992) ilustra la cuestión con el ejemplo de los isómeros químicos, como el orto- y el para-diclorobenceno: si cada uno de ambos tipos de moléculas tiene una forma definida por su vector de estado en el mismo espacio de Hilbert, su superposición también es un estado posible según la mecánica cuántica. Frente a ello el autor se pregunta: “¿Hay alguna razón apropiada para excluir tales superposiciones? ¿Qué razón puede darse —desde un punto de vista mecánico-cuántico - en favor de la sistemática química?" (Amann 1992, p.32).

En definitiva, las moléculas de las que se ocupa la química molecular son objetos sustanciales y locales, individuos en términos filosóficos, "que existen separada e independientemente en el sentido de que puede decirse consistentemente que 'tienen' ciertas propiedades, sea que interactúen o no con alguna otra cosa" (Wooley 1978, p.1074). En el contexto de esta ontología clásica, es razonable que los átomos componentes de la molécula sean concebidos en términos compatibles con dicha ontología. Si bien los electrones ya no giran en órbitas definidas en torno a los núcleos, sigue 
hablándose de la probabilidad de que los electrones se encuentren en una región del espacio en torno al núcleo. En otras palabras, la molécula es concebida como un objeto compuesto de núcleos clásicos y electrones semi-clásicos, cuya "semi" clasicidad se debe exclusivamente a que no se encuentran regidos por las ecuaciones clásicas de movimiento sino por la ecuación de Schrödinger bajo su interpretación probabilística. Un átomo es, entonces, un objeto individual - el núcleo- espacialmente localizado, con otros objetos individuales - los electrones- que se disponen a su alrededor de un modo que la teoría determina sólo probabilísticamente. Es claro que esta imagen del átomo es totalmente ajena a la mecánica cuántica, donde estrictamente sólo podría hablarse del sistema-molécula, descripto por su función de onda no-separable, con sus correlaciones cuánticas con los restantes sistemas con los que ha interactuado, y cuyos "componentes" no son los objetos individuales sustanciales y locales- de la física clásica.

Las estrategias tradicionales para conectar la descripción química y la descripción cuántica de los sistemas moleculares se basan en la introducción de la conocida aproximación de Born-Oppenheimer, basada en la separación de los movimientos del núcleo y de los electrones. La aproximación procede en dos pasos: en un primer paso se ignora el movimiento de los núcleos, despreciando su energía cinética, y se resuelve la ecuación de Schrödinger sólo para los electrones; en un segundo paso se reintroduce la energía cinética de los núcleos. Esta técnica brinda resultados aceptables bajo el supuesto de que los núcleos se mueven lentamente respecto de los electrones, lo cual a su vez se basa en el hecho de que la masa $M$ del núcleo es muchísimo mayor que la masa $m$ de los electrones. Sin embargo, desde un punto de vista formal, la aproximación de Born-Oppenheimer es una expansión asintótica en torno al límite singular $\varepsilon=m / M \rightarrow 0$. Esto significa que el nexo entre la descripción química y la descripción cuántica sería estrictamente válido sólo para masas nucleares infinitas; únicamente en este caso "las correlaciones holísticas entre los núcleos y los electrones quedan suprimidas y la descripción de una molécula se reduce a la descripción del movimiento de electrones en el campo eléctrico de un núcleo clásico" (Primas 1998, p.91). Se trata, entonces, de un límite singular, que implica "un cambio cualitativo en la teoría, el cual se expresa en la teoría por una aproximación discontinua" (Wooley 1978, p.1076). Como Wooley y Primas señalan, en muchos sentidos la aproximación de Born-Oppenheimer es análoga a la aproximación semi-clásica WKB en mecánica cuántica con su correspondiente límite singular $\hbar \rightarrow 0$. Tal analogía no resulta sorprendente cuando la cuestión se encara desde una perspectiva más general: ambas aproximaciones son respuestas parciales al problema general y aún irresuelto del límite clásico de la mecánica cuántica, esto es, el problema de explicar cómo surgen los objetos individuales y locales de la ciencia clásica - no-cuántica - a partir de la descripción holista y no-sustancial de la mecánica cuántica.

En definitiva, la química molecular trabaja con moléculas clásicas, espacialmente

Principia 14(3): 309-333 (2010). 
localizadas, sin superposiciones ni correlaciones, compuestas de átomos descriptos en términos de núcleos clásicos y electrones semi-clásicos; la forma de las regiones ocupadas por tales electrones con mayor probabilidad - los orbitales- explica la forma de las moléculas, forma que, a su vez, explica muchas de las propiedades de las sustancias. Como hemos señalado, actualmente muchos autores admiten no sólo la no reductibilidad de la descripción química a la mecánica cuántica, sino la discontinuidad conceptual entre ambos ámbitos: los conceptos que utiliza la química molecular se encuentran totalmente ausentes del universo conceptual de la mecánica cuántica. La cuestión es, ahora, decidir la posición a adoptar frente a esta ruptura conceptual.

\section{El supuesto de una ontología única}

Cuando nos desembarazamos de las consideraciones acerca de limitaciones computacionales y reconocemos que el problema de los orbitales es un problema conceptual que se presenta en cualquier tipo de átomo, sea mono o multielectrónico, la ruptura se hace evidente. En mecánica cuántica el concepto de orbital refiere a la función de onda del átomo que, en tanto entidad matemática, no habita el mundo cuántico. En química molecular, el concepto de orbital refiere a una región del espacio donde se encuentran con mayor probabilidad los electrones semi-clásicos en torno del núcleo clásico. En otras palabras, los orbitales no existen en el mundo descripto por la mecánica cuántica pero sí existen en el mundo descripto por la química molecular. ¿Por qué, entonces, se concluye que, sencillamente, los orbitales no existen?

Consideremos dos teorías $T_{1}$ y $T_{2}$ tales que $T_{2}$ contiene un concepto referencial $C$ que en $T_{1}$ no refiere. La entidad referida por $C$ no existe simpliciter sólo si suponemos que $T_{1}$ es la teoría "verdadera" o, al menos, la más adecuada para describir la realidad. Este supuesto está implícito en las palabras de Scerri cuando sostiene que "es esencial que los científicos sean más cuidadosos al atribuir realidad física a entidades definidas teóricamente y respecto de las cuales la teoría nos informa que no existen físicamente" (Scerri 2001, p, S81). ¿Cuál es la teoría a la que se refiere el autor, que nos informa que los orbitales no existen? La mecánica cuántica, obviamente. Pero, ¿por qué no preguntarle a la química molecular? ¿Qué privilegio metafísico detenta la mecánica cuántica para constituirse en el testigo clave acerca de lo que existe o no existe en el mundo? Es claro que el prestigio se funda en el supuesto ontológicamente reduccionista según el cual existe una ontología única, y la mecánica cuántica es la mejor teoría para describir dicha ontología: toda descripción que no concuerde con la descripción cuántica estará irremediablemente confinada al ámbito de lo fenomenológico y no-referencial.

Por supuesto, nada tiene de contradictorio desde un punto de vista lógico la adopción de un reduccionismo ontológico como el que conduce a negar la existencia

Principia 14(3): 309-333 (2010). 
de los orbitales. Pero es interesante reflexionar acerca de las consecuencias que conlleva una defensa consistente de tal postura. Si la mecánica cuántica fuera la única teoría verdadera (o aproximadamente verdadera) ya que es la única que describe (al menos aproximadamente) la realidad tal como es en sí misma, no sólo los orbitales no existirían, sino tampoco todas las entidades de las que nos hablan otras teorías y disciplinas científicas. Se esfumarían, así, las moléculas con su forma molecular, los gases con sus temperaturas, los planetas con sus trayectorias, los organismos biológicos con sus células, y así siguiendo. Si la observación de orbitales implicara "una rotunda refutación de la mecánica cuántica" (Scerri 2001, p.S76), la observación de cualquier otra entidad que no pertenece al dominio ontológico de la mecánica cuántica también la refutaría; sin embargo, toda la ciencia no-cuántica se respalda en tales observaciones. Según Scerri, puesto que el término 'orbital' es no referencial en la mecánica cuántica, los orbitales no existen y, por tanto, "no pueden ser observados, punto" (Scerri 2000, p.1494). Reemplacemos en esta tesis el término 'orbital' por cualquier otro término científico no perteneciente al lenguaje de la mecánica cuántica - 'molécula', 'célula', 'planeta', 'virus', 'galaxia', 'péndulo'— : un reduccionismo coherente debería asentir también a la afirmación resultante de tal reemplazo.

Como vemos, el debate en torno a los orbitales, difundido y amplificado por la resonancia de ciertos resultados experimentales, es sólo una manifestación particular del viejo problema de la reducción ontológica: admitir la existencia de una única ontología "real" conduce necesariamente a confinar al ámbito de lo inexistente o de la mera apariencia todo aquello que no habita esa ontología, aun cuando hablemos de ello en otras teorías científicas exitosas e incluso en nuestro discurso cotidiano. Afortunadamente, no es ésta la única alternativa filosófica.

\section{En favor de un pluralismo ontológico pragmático}

Como señalamos en la Sección 2, en trabajos previos (Lombardi y Labarca 2004, 2005) hemos defendido un pluralismo ontológico de raigambre kantiana que nos ha permitido admitir la existencia igualmente "real" de diferentes ontologías, sin relaciones de prioridad o dependencia entre ellas. Desde esta perspectiva, puesto que toda ontología surge de la síntesis entre realidad nouménica y esquema conceptual, el dominio ontológico de la cuántica resulta tan constituido como el de la química molecular y, en consecuencia, no cuenta con privilegio alguno. Por lo tanto, puede afirmarse de un modo consistente que los orbitales, la forma molecular y los enlaces químicos no existen en la ontología cuántica pero existen en la ontología de la química molecular: el mundo de la química preserva su autonomía ontológica puesto que las entidades químicas no necesitan de las entidades cuánticas para legitimar su existencia.

Principia 14(3): 309-333 (2010). 
Frente a esta postura filosófica, el reduccionista ontológico suele apelar a los nexos interteóricos que vinculan diferentes teorías como argumento en favor de la reducción: la temperatura no es más que energía cinética media por molécula puesto que, si así no fuera, la igualdad entre sus valores sería un milagro. Siguiendo esta línea argumentativa, la ciencia avanzaría hacia una unificación reductiva, y cada nuevo caso exitoso de relación interteórica sería una nueva prueba de la existencia de una ontología fundamental cuyas leyes explicarían, en principio, todo aquello que observamos. Este argumento, sin embargo, suele apoyarse en casos de relación interteórica altamente idealizados, pasando por alto las dificultades de vincular teorías diferentes en situaciones donde la complejidad de los sistemas impide simplificaciones y hace inadecuadas las aproximaciones. En el caso de la termodinámica, la temperatura $T$ puede calcularse en términos de la energía cinética media por molécula $\bar{E}_{K}$ sólo en el caso de gases ideales, donde se ignoran las interacciones entre partículas; en gases reales, plasmas o sólidos, la famosa igualdad deja de ser válida. Pero aún en los casos en que pueda afirmarse con suficiente aproximación que $T=(2 / 3 k) \bar{E}_{K}$, la expresión sólo indica la igualdad entre los valores numéricos de las dos magnitudes físicas. El hecho de que el valor numérico de la temperatura coincida con el de la energía cinética media (salvo constante) no identifica los referentes de los términos ' $T$ ' y ' $\bar{E}_{K}$ ' (cfr. Lombardi y Pérez Ransanz 2011a). Se trata de conceptos diferentes que adquieren sus significados en contextos teóricos y empíricos diferentes: la temperatura es una propiedad que se define y mide con los recursos teóricos y experimentales de la termodinámica, y para ello no necesita relacionarse con otra propiedad definida en el contexto teórico de la mecánica.

Si la reducción ontológica, con su identificación de entidades pertenecientes a dominios diferentes, resulta cuestionable en el caso más sencillo de relación interteórica, su plausibilidad se debilita aún más en el caso de la relación entre química molecular y mecánica cuántica. En este caso, ambas teorías no se vinculan mediante sencillas relaciones funcionales como en el caso $T=(2 / 3) \bar{E}_{K}=f\left(\bar{E}_{K}\right)$, sino que en sus nexos intervienen operaciones de límite matemático como en la aproximación de Born-Oppenheimer. Bien sabido es que la operación matemática de 'paso al límite' no equivale ni puede expresarse como una función y, por tanto, introduce una heterogeneidad ya en el propio nivel matemático: el número que se obtiene de un límite puede ser de una naturaleza completamente diferente a la de los números de la sucesión de la cual es límite. Como bien afirma Rohrlich, el nexo entre dos teorías a través de un límite matemático introduce una ruptura conceptual que no brinda elemento alguno en favor de la reducción ontológica; por el contrario, los conceptos así vinculados denotan entidades pertenecientes a ontologías diferentes, y "las ontologías de teorías en niveles diferentes son inconmensurables" (Rohrlich 2001, p.200; énfasis del autor). En particular, trabajos recientes muestran el modo en que nuevos y complejos fenómenos surgen en las fronteras asintóticas entre teorías relacionadas 
por límites singulares (cfr. Batterman 2002). En el caso de la química molecular, Primas ha insistido sistemáticamente sobre este punto: por ejemplo, la forma molecular es un concepto que sólo puede obtenerse de la mecánica cuántica "como una estructura asintótica en el límite singular para masas moleculares infinitas" y, por ello, es un "concepto clásico" que "puede discutirse usando un lenguaje booleano irrestricto" ajeno a la mecánica cuántica (Primas 1983, p.335).

En definitiva, no hay argumentos formales, basados en las relaciones matemáticas entre teorías diferentes, que respalden la idea de una reducción de un dominio ontológico respecto de otro. Pero si el formalismo no brinda apoyo al reduccionista en la defensa de su tesis, la práctica efectiva de la ciencia tampoco viene en su ayuda. La química molecular ha desarrollado un vasto edificio teórico, basado en conceptos irreductibles a la mecánica cuántica, mediante el cual ha logrado explicar un sinnúmero de fenómenos observables y reproducibles en el laboratorio. Conceptos como orbital atómico, enlace químico y forma molecular no sólo han sido empíricamente exitosos en la práctica de la disciplina, sino que han resultado extremadamente fructíferos en la manipulación y obtención de diferentes sustancias químicas, tanto naturales como artificiales. Lo que convierte a la química molecular en una disciplina pujante nada tiene que ver con sus relaciones con la mecánica cuántica: su valor científico se funda en el éxito de sus múltiples aplicaciones tecnológicas. Frente a este hecho innegable, no parece razonable disolver las entidades químicas en la inexistencia como meras "metáforas" (Wooley 1982, p.4) que, al vincularse con la "verdadera" realidad cuántica, desaparecen "en el aire" (Wooley 1978, p.1077). Como afirma Hacking (1983), hemos reflexionado demasiado acerca del plano de la representación, descuidando la intervención en la práctica efectiva de la ciencia; es en este ámbito donde debemos encontrar el criterio de existencia de las entidades de las que nos hablan nuestras teorías. Admitimos la existencia de entidades inobservables, como los electrones, cuando podemos "rociarlos" ("spray them") (Hacking 1983, p.23), es decir, cuando podemos utilizar sus propiedades para intervenir en otros aspectos de la naturaleza: "Estamos completamente convencidos de la realidad de los electrones cuando nos proponemos construir-y tenemos suficiente éxito en construir- nuevos tipos de dispositivos que usan las diversas y bien conocidas propiedades causales de los electrones para interferir en otras partes de la naturaleza más hipotéticas" (Hacking 1983, p.265). Esto significa que, en opinión de Hacking, la existencia de entidades inobservables queda establecida cuando se las utiliza en la producción de nuevos fenómenos bajo el supuesto de que contribuyen de un modo esencial en dicha producción.

Si atendemos, entonces, al cambio de perspectiva que nos propone Hacking, nuestras conclusiones ontológicas acerca del dominio referido por la química molecular deben dejar de basarse en consideraciones formales acerca de relaciones interteóricas, para tomar en cuenta la manipulación de entidades químicas en la práctica

Principia 14(3): 309-333 (2010). 
tecnológica. Cuando adoptamos esta perspectiva pragmática, la química molecular lleva las de ganar: su asombrosa fertilidad en la manipulación de sustancias conocidas y en la creación de nuevas sustancias se convierte en el mejor argumento en favor de la existencia de las entidades que pueblan su ontología.

Supongamos por un momento que en un futuro próximo la mecánica cuántica fuera abandonada en el ámbito de la física debido a, por ejemplo, nuevos fenómenos de los cuales no puede dar cuenta. Tal situación representaría ciertamente una profunda conmoción en la comunidad de los físicos cuánticos, quienes se verían obligados a reformular sus descripciones en términos de la nueva teoría que viniera a reemplazar a la cuántica original. Por el contrario, la química molecular no se vería afectada del mismo modo; como afirma van Brakel, "Si la mecánica cuántica resultara equivocada, esto no afectaría todo (o incluso ningún) conocimiento químico acerca de las moléculas (unión, estructura, valencia, etc.). Si la química molecular resultara equivocada, esto no descalificaría todo (o incluso ningún) conocimiento acerca, digamos, del agua" (van Brakel 2000b, p.177). Así como la práctica empírica de la termodinámica se mantuvo inmune frente al cambio de la teoría supuestamente fundamental, que pasó de la teoría del calórico a la mecánica clásica y finalmente a la mecánica cuántica, las múltiples aplicaciones prácticas de la química molecular no desaparecerían con el abandono de la mecánica cuántica, y éste es el mejor argumento en favor de la existencia de las entidades de las que nos habla la teoría. Cuando nos libramos del supuesto metafísico de una realidad única descripta por la "Teoría Verdadera", podemos aceptar la coexistencia de múltiples ontologías autónomas, cuyo carácter "real" se funda en el éxito de nuestra interacción con las entidades que la conforman. Los orbitales atómicos son un ejemplo de estas entidades.

\section{Conclusiones}

A modo de conclusión, formulemos claramente las preguntas sobre las que gira el debate acerca de los orbitales atómicos, y brindemos sus respuestas precisas desde nuestra perspectiva. ¿Existen los orbitales atómicos? Existen en la ontología de la química molecular, pero no existen en la ontología cuántica. Esta respuesta resulta no contradictoria desde un pluralismo ontológico que admite la coexistencia de diferentes ontologías autónomas, sin prioridades ni dependencias mutuas. ¿Los orbitales pueden ser observados? Sí, pueden ser observados como pueden serlo otras entidades no pertenecientes a la ontología cuántica. Esto no significa abrir un juicio acerca del particular proceso experimental utilizado en los anuncios acerca de la "visualización de orbitales": sólo los experimentalistas especializados están en condiciones de analizar los detalles del experimento y evaluar su fiabilidad (para detalles, cfr. Wang y Schwarz 2000a,b, Zuo et al. 2000). Nuestra respuesta se refiere exclusivamente al

Principia 14(3): 309-333 (2010). 
hecho de que, en principio, no existe razón alguna que impida la observación de entidades que no pertenecen al dominio cuántico.

Sin duda, esta posición se enfrenta a los supuestos metafísicos reduccionistas que, explícita o implícitamente, continúan impregnando las opiniones que predominan en el ámbito científico e incluso en el contexto de la filosofía de la química. Sin embargo, cuando estos supuestos se traen a la luz para someterlos a un cuidadoso análisis, los argumentos en su favor distan de ser concluyentes. Por ello es que adoptamos un marco filosófico ontológicamente pluralista, que no nos compromete con las difícilmente admisibles conclusiones del reduccionismo ontológico tradicional. Y el caso de los orbitales atómicos no se ha presentado como un obstáculo para ello. ${ }^{1,2}$

\section{Referencias}

Amann, A. 1992. Must a Molecule Have a Shape? South African Journal of Chemistry 45: 29-38.

Bader, R. F. W. 2003. Letter to the Editor: Quantum Mechanics or Orbitals? International Journal of Quantum Chemistry 94: 173-77.

Batterman, R. 2002. The Devil in the Details. Asymptotic Reasoning in Explanation, Reduction, and Emergence. Oxford: Oxford University Press.

Benfey, T. 2000. Reflections on the Philosophy of Chemistry and a Rallying Call for Our Discipline. Foundations of Chemistry 2: 195-205.

Bishop, R. 2005. Patching Physics and Chemistry Together. Philosophy of Science 72: 710-22.

Broad, C. D. 1925. The Mind and Its Place in Nature. London: Routledge and Kegan Paul.

Bunge, M. 1982. Is Chemistry a Branch of Physics?. Zeitschrift fur allgemeine Wissenschaftstheorie 13: 209-23.

Crasnow, S. 2000. How Natural Can Ontology Be?. Philosophy of Science 67: 114-32.

Dirac, P. A. M. 1929. Quantum Mechanics of Many-Electron Systems. Proceedings of the Royal Society A338: 714-33.

Emerson, K. 1999. The Quantum Mechanical Explanation of the Periodic System. Journal of Chemical Education 76: 1189.

Hacking, I. 1983. Representing and Intervening. Cambridge: Cambridge University Press; v.e. Representar e Intervenir. México: Coedición Paidós-UNAM 1996.

Hendry, R. 2006. Is There Downward Causation in Chemistry?. In: Baird, D., Scerri, E. and McIntyre, L. (eds.) Philosophy of Chemistry: Synthesis of a New Discipline. The Netherlands: Boston Studies in the Philosophy of Science, Springer.

Humphreys, C. J. 1999. Electron Seen in Orbit. Nature 401: 49-52.

Itatani, J., Levesque, J., Zeidler, D., Niikura, H., Pépin, H., Kieffer, J. C., Corkum, P. B. y Villeneuve, D. M. 2004. Tomographic Imaging of Molecular Orbitals. Nature 432: 86771.

Jacoby, M. 1999. Picture-Perfect Orbitals. Chemical \& Engineering News 77: 8.

Jenkins, Z. 2003. Do You Need to Believe in Orbitals to Use Them?: Realism and the Autonomy of Chemistry. Philosophy of Science 70: 1052-62.

Kemeney, J. G. and Oppenheim, P. 1956. On Reduction. Philosophical Studies 7: 6-19.

Principia 14(3): 309-333 (2010). 
Lombardi, O. y Labarca, M. 2004. En Defensa de la Autonomía Ontológica del Mundo Químico. Diálogos 84: 51-70.

- 2005. The Ontological Autonomy of the Chemical World. Foundations of Chemistry 7: $125-48$.

- 2006. The Ontological Autonomy of the Chemical World: A Response to Needham. Foundations of Chemistry 8: 81-92.

Lombardi, O. y Pérez Ransanz, A. R. 2011a. Lenguaje, ontología y relaciones interteóricas: en favor de un genuino pluralismo ontológico. Revista Arbor. Ciencia, Pensamiento y Cultura 187: 43-52.

- 2011b. Los Múltiples Mundos de la Ciencia. Un Realismo Pluralista y su Aplicación a la Filosofía de la Física. México: UNAM-Siglo XXI, en prensa.

Luisi, P. L. 2002. Emergence in Chemistry: Chemistry as the Embodiment of Emergence. Foundations of Chemistry 4: 183-200.

Mclntyre, L. 2007. The philosophy of chemistry: ten years later. Synthese 155: 291-92.

Needham, P. 2006. Ontological Reduction: A Comment on Lombardi and Labarca. Foundations of Chemistry 8: 73-80.

Nye, M. J. 1993. From Chemical Philosophy to Theoretical Chemistry - Dynamics of Matter and Dynamics of Disciplines 1800-1950. Berkeley: University of California Press.

Pascual, J. I., Gómez-Herrero, J., Rogero, C., Baró, A. M., Sánchez-Portal, D., Artacho, E., Ordejón, P. y Soler, J. M. 2000. Seeing Molecular Orbitals. Chemical Physical Letters 321: 78-82.

Primas, H. 1983. Chemistry, Quantum Mechanics and Reductionism. Berlin: Springer.

- 1987. Contextual Quantum Objects and their Ontic Interpretation. In Lathi, P. and Mittelstaedt, P. (eds.) Symposium on the Foundations of Modern Physics 1987. Singapore: World Scientific.

- 1994. Hierarchic Quantum Descriptions and their Associated Ontologies. In Laurikainen, K. V. Montonen, C. and Sunnarborg, K. (eds.) Symposium on the Foundations of Modern Physics 1994. Gif-sur-Yvette: Editions Frontières.

— 1998. Emergence in Exact Natural Sciences. In Farre, G. and Oksala, T. (eds.) Acta Polytechnica Scandinavica 91: 83-98.

Richman, R. 1999a. In Defense of Quantum Numbers. Journal of Chemical Education 76: 608.

— 1999b. The Use of One-Electron Quantum Numbers to Describe Polyatomic Systems. Foundations of Chemistry 1: 175-83.

Rohrlich, F. 2001. Cognitive Scientific Realism. Philosophy of Science 68: 185-202.

Scerri, E. R. 2000a. Realism, Reduction and the 'Intermediate Position'. In Bhushan, N. and Rosenfeld, S. (eds.) Of Minds and Molecules. New Philosophical Perspectives on Chemistry. New York: Oxford University Press.

- 2000b. Have Orbitals Really Been Observed?. Journal of Chemical Education 77: 1492-94.

- 2001. The Recently Claimed Observation of Atomic Orbitals and Some Related Philosophical Issues. Philosophy of Science 68: S76-S88.

- 2004. Just How Ab Initio is Ab Initio Quantum Chemistry?. Foundations of Chemistry 6: $93-116$.

- 2005. Editorial 20. Foundations of Chemistry 7: 119-23.

Principia 14(3): 309-333 (2010). 
2006. Normative and Descriptive Philosophy of Science and the Role of Chemistry. In: Baird, D., Scerri, E. and McIntyre, L. (eds.) Philosophy of Chemistry: Synthesis of a New Discipline. The Netherlands: Boston Studies in the Philosophy of Science, Springer.

- 2007. The Periodic Table - Its Story and Its Significance. New York: Oxford University Press.

Scerri, E. R. and McIntyre, L. 1997. The Case for the Philosophy of Chemistry. Synthese 111: 213-232.

Spence, J. C.; O'Keefe, M. and Zuo, J. M. 2001. Have Orbitals Really Been Observed?. Journal of Chemical Education 78: 877.

Tinoco Jr. I.; Sauer, K.; Wang, J.C.; Puglisi, J.D. 2002. Physical Chemistry - Principles and Applications in Biological Sciences. New Jersey: Prentice Hall.

van Brakel, J. 2000a. Philosophy of Chemistry. Between the Manifest and the Scientific Image, Leuven: Leuven University Press.

- 2000b. The Nature of Chemical Substances. In Bhushan, N. and Rosenfeld, S. (eds.) Of Minds and Molecules. New Philosophical Perspectives on Chemistry. New York: Oxford University Press.

- 2003. The Ignis Fatuus of Reduction and Unification. Back to the Rough Ground. Annals of New York Academy of Sciences 988: 30-43.

Vancik, H. 1999. Opus Magnum: An Outline for the Philosophy of Chemistry. Foundations of Chemistry 1: 242-56.

Vemulapalli, G. K. and Byerly, H. 1999. Remnants of Reductionism. Foundations of Chemistry 1: 17-41.

Wang, S. G. and Schwarz, W. H. 2000a. On Closed-Shell Interactions, Polar Covalences, d Shell Holes, and Direct Images of Orbitals: The Case of Cuprite. Angewandte Chemie International Edition 39: 1757-62.

- 2000b. Final Comment on the Discussions of 'The Case of Cuprite'. Angewandte Chemie International Edition 39: 3794-96.

Wasserman, E. and Schaefer, H.F. 1986. "Methylene Geometry." Science 233: 829.

Wooley, R. G. 1978. Must a Molecule Have a Shape?. American Chemical Society 100: 107378.

- 1982. Natural Optical Activity and the Molecular Hypothesis. Structure and Bonding 52: 1-35.

Yam, P. 1999. Seeing the Bonds. Scientific American 281: 28.

Zuo, J. M., Kim, M., O'Keefe, M. and Spence, J. C. H. 1999. Direct Observation of d-Orbital Holes and Cu-Cu Bonding in $\mathrm{Cu}_{2} \mathrm{O}$. Nature 401: 49-52.

- 2000. On Closed-Shell Interactions, Polar Covalences, d Shell Holes, and Direct Images of Orbitals: The Case of Cuprite. Response to the Essay by S. G. Wang and W. H. E. Schwarz. Angewandte Chemie International Edition 39: 3791-94.

Zurer, P. 1999. Chemistry's Top Five Achievements. Chemical \& Engineering News 77: 38-40.

MARTín LABARCA

Consejo Nacional de Investigaciones Científicas y Técnicas (CONICET)

Universidad Nacional de Quilmes

Buenos Aires

ARGENTINA

mglabarca@unq.edu.ar

Principia 14(3): 309-333 (2010). 
Olimpia LOMBARDi

Consejo Nacional de Investigaciones Científicas y Técnicas (CONICET)

Universidad de Buenos Aires

Buenos Aires

ARgENTINA

olimpiafilo@arnet.com.ar

\section{Notas}

${ }^{1}$ Una versión preliminar de este trabajo fue presentada en el $V$ Encuentro de Filosofía $e$ Historia de la Ciencia del Cono Sur, Asociación de Filosofía e Historia de la Ciencia del Cono Sur (AFHIC), Florianópolis, Brasil, 22 al 25 de Mayo de 2006.

${ }^{2}$ Agradecimientos: A uno de los referees anónimos por sus sugerencias. Este trabajo fue posible gracias al apoyo brindado por el Consejo Nacional de Investigaciones Científicas y Técnicas (CONICET), la Agencia Nacional de Promoción Científica y Tecnológica (FONCyT) y la Sociedad Argentina de Análisis Filosófico de Argentina.

Principia 14(3): 309-333 (2010). 